\title{
Entorno educativo y aprendizaje, estrategias de estudio en el idioma ingles
}

\section{Educational environment and learning, study strategies in the English language}

Esthela Isabel Colcha Guashpa. ${ }^{1}$, Zoila Victoria Herrera Andrade. ${ }^{2}$, Rocío de los Ángeles Barragán Murillo. ${ }^{3}$, Daniela Fernanda Guano. ${ }^{4}$ \& Edison Hernán Salazar Calderón. ${ }^{5}$

\begin{abstract}
DOI: https://doi.org/10.33262/cienciadigital.v3i2.6.571

The process of learning English as a foreign language is generally given within the classroom, where different controlled activities are carried out, although this process is mostly in the educational field, students can reach a high English language development standard, because they have opportunity to learn during their training, and scarcely in other types of activities, however the student is limited to communicate in this language in some occasions because it is practiced outside the classroom a few times. Despite this limitation, the learning of the English language is related to the revision of the basic bibliography of the different careers and the information on the internet which is in English becoming university education the last step towards professionalization, this importance is given from the school point of view, so that students can dominate English language. The importance of learning English as a foreign language is vital for the correct performance of the teaching process in order to get a quality education to all, to support students contributing to their well-being in which they can reach their full potential, where it is determined by various physical, psychological and social factors. When we talk about designing the educational environment, it not only consists on organizing the space, but also on organizing carefully the time for the different activities, the materials to be used, the characteristics of the students according to their ages and the proposed activities should not vary significantly from their family and cultural environment, as well as their psycho-evolutionary characteristics.
\end{abstract}

Keywords: Educational environment, Learning, Study strategies, English language

\footnotetext{
${ }^{1}$ Escuela Superior Politécnica de Chimborazo, Chimborazo, Ecuador, e_colcha@espoch.edu.ec

${ }^{2}$ Escuela Superior Politécnica de Chimborazo, Chimborazo, Ecuador, zoila.herrera@espoch.edu.ec

${ }^{3}$ Escuela Superior Politécnica de Chimborazo, Chimborazo, Ecuador, robarragan@espoch.edu.ec

${ }^{4}$ Escuela Superior Politécnica de Chimborazo, Chimborazo, Ecuador, daniela.guano@espoch.edu.ec

${ }^{5}$ Escuela Superior Politécnica de Chimborazo, Chimborazo, Ecuador, edisonh.salazar@espoch.edu.ec
} 


\section{Resumen}

El proceso de aprendizaje del inglés como lengua extranjera generalmente se da dentro del aula de clase, lugar en el cual se realizan diferentes actividades de tipo controladas, pese a que este proceso se da en su mayor parte en el ámbito educativo, los estudiantes pueden alcanzar un alto grado de desarrollo del idioma inglés, al tener la oportunidad de aprender durante su formación, y escasamente en otro tipo de actividades, sin embargo limita en ciertas ocasiones a que el estudiante pueda finalmente ser capaz de comunicarse en este idioma, dado que se reducen el número de veces de la práctica del idioma fuera del aula de clases. Pese a este limitante el aprendizaje del idioma inglés, se relaciona con la revisión de bibliografía básica que se encuentra en inglés de las distintas carreras, así como la información expuesta en internet; constituyéndose la enseñanza universitaria en el último peldaño hacia la profesionalización, relevancia que se da desde el punto de vista escolar para que los estudiantes alcancen un correcto dominio de este idioma. La importancia que tiene el aprendizaje del inglés como lengua extranjera es vital para el desempeño correcto del proceso de enseñanza para una educación de calidad, accesible a todos; delineado para apoyar a los estudiantes contribuyendo a su bienestar en el que pueden alcanzar su pleno potencial, en donde está determinada por varios factores físicos, psicológicos y sociales. Cuando hablamos de diseñar el entorno educativo, no sólo consiste en organizar el espacio, sino también en diseñar cuidadosamente el tiempo para las diferentes actividades, los materiales que vamos a utilizar, las características de nuestros estudiantes considerando sus edades y que las actividades que planteemos no varíen significativamente de su entorno familiar y cultural, así como sus características psicoevolutivas.

Palabras clave: Entorno educativo, Aprendizaje, Estrategias de estudio, Idioma ingles

\section{Introducción}

En la última década se ha dado importancia al aprendizaje del idioma inglés como lengua extranjera a una edad temprana en la mayoría de establecimientos educativos contribuyendo numerosos estudios a esta expansión; al asegurar que los niños aprenden segundas lenguas a una edad temprana, desarrollan sus sistemas gramaticales de manera no consciente y natural, por el hecho de entrar en contacto con los datos lingüísticos de la lengua en cuestión.

En la actualidad el idioma inglés se considera uno de los activos más importantes del conocimiento dentro de la esfera social, por cuanto en la sociedad moderna se han originado nuevos campos del saber en el orden tecnológico, comercial y cultural donde el inglés abarca significativamente nuevos espacios creando un liderazgo imprescindible en el mercado nacional e internacional.

El aprendizaje del idioma ingles se vale de varias técnicas o métodos que permiten un comunicativo de enseñanza para transmitir el conocimiento y enseñar gramática, vocabulario, expresión oral, comprensión auditiva, lectura y escritura, con el propósito de mejorar la 
capacidad de comunicación mediante técnicas en las que el profesor actúa como facilitador en la clase, mientras alienta a los estudiantes a utilizar la lengua inglesa todo el tiempo posible.

El vocabulario es una parte importante del aprendizaje y la comprensión de toda lengua. Los estudiantes están rodeados de vocabulario en inglés todos los días en la vida real, ya sea en películas, medios de comunicación y la vida diaria, mismo que se acrecienta con el tiempo y la elaboración de listas de términos extraídos de fuentes como libros u otros textos y materiales. La tarea del profesor es asegurarse de que los estudiantes aprendan y comprendan todo este vocabulario, además se puede reafirmar el nuevo vocabulario adquirido a través de diversos ejercicios de comprensión que contengan las palabras desconocidas; estos ejercicios también pueden servir para ayudar a los estudiantes a aprender y retener las nuevas palabras, mediante técnicas de aprendizaje como ejercicios de vocabulario, búsqueda de palabras, pruebas y juegos.

Se debe considerar técnicas de enseñanza para la comprensión auditiva del inglés debiendo incluir la participación, demostración y colaboración de los estudiantes en clase animándolos a hablar con confianza y utilizar las estructuras gramaticales correctas para que puedan entender, así también es fundamental que comprendan lo que están escuchando para que puedan responder adecuadamente. (Fontana, 1994)

Tanto el rol del docente como del estudiante constituye un papel esencial en el aprendizaje del idioma inglés, pese a la importancia que están adquiriendo los nuevos métodos, el papel del docente sigue siendo muy relevante, como afirma Nunan (1989: 134) "la variable crucial en la situación de enseñanza son los profesores"

Por tal razón es necesario incluir la enseñanza del inglés desde una Pedagogía que abarque estrategias innovadoras y contribuyan a la actividad formativa basándose en la sensibilidad y comprensión humana, incentivando y estimulando a los estudiantes para construir sus aprendizajes, en un ambiente alegre, motivador y empático con el fin de fortalecer el desarrollo de las capacidades y destrezas lingüísticas del idioma.

\section{Entorno educativo}

La autora López (2005) en su libro Modelo de teoría y diseños didácticos, al hablar de entorno o ambiente educativo, se refiere al espacio organizado y estructurado de tal manera que facilite el acceso al conocimiento de actividades reales y con motivos de aprendizaje. Permitiendo no solamente abordar la realidad del mundo material y transformarlo sino intercambiar la información y comunicación con otros. Requiere de una participación activa en el proceso como condición necesaria para lograr interacción e interactividad, constituyéndose en formas básicas de participación donde los ambientes de aprendizaje exigen del maestro la planeación previa, una guía de orientación alumno hacia un logro de competencia, la contextualización de lo que se aprende, así como la creación en un ambiente de comunicación. 
(Freire, 1980), expone que la educación no es tan sólo un "relato" de enseñanza ejercida unidireccionalmente y en sentido vertical, sino sobre todo una indagación participativa sobre la realidad de los propios sujetos, que, mediante la propia investigación, ganan conciencia sobre sí y sobre su mundo para transformarlo. "Es por esto - afirma - que la investigación se hará tanto más pedagógica cuanto más crítica y tanto más crítica en cuanto, dejando de perderse en los esquemas estrechos de las visiones parciales de la realidad, de las visiones 'focalistas' de la realidad, se fije en la comprensión de la totalidad" (p.133).

La expresión ambiente educativo induce a pensar el ambiente como sujeto que actúa con el ser humano y lo transforma. De allí se deriva que educa la ciudad (la ciudad educadora), la calle, la escuela, la familia, el barrio y los grupos de pares, entre otros. Reflexionar sobre ambientes educativos para el sano desarrollo de los sujetos convoca a concebir un gran tejido construido con el fin específico de aprender y educarse.

\section{Componentes en un ambiente educativo}

(López, 2005) Plantea dos componentes en todo ambiente educativo: los desafíos y las identidades.

- Los desafíos, entendidos como los retos y las provocaciones que se generan desde las iniciativas propias o las incorporadas por promotores, educadores y facilitadores, entre otros. Son desafíos en tanto son significativos para el grupo o la persona que los enfrenta, y con la menor intervención de agentes externos. Los desafíos educativos fortalecen un proceso de autonomía en el grupo y propician el desarrollo de los valores.

- Los ambientes educativos también están signados por la identidad, pues la gestión de las identidades y lo cultural propio es la posibilidad de creación de relaciones de solidaridad, comprensión y apoyo mutuo e interacción social.

En líneas generales, la comunidad educativa se encarga de promover actividades que lleven al mejoramiento de la calidad de la educación, y lograr además mejorar el bienestar psicosocial de los estudiantes. (Rojas, 2016)

\section{El ambiente de aprendizaje desde distintas perspectivas}

Los autores Loughlin (Duval, P, \& Vayer, R. , 1993)y Suina (2002), en su libro Ambiente de aprendizaje, exponen que existen varios ambientes con características y las implicaciones que estas conllevan sobre los individuos de una comunidad educativa, citando a continuación:

- El ambiente de aprendizaje como espacio físico - sensorial

- El ambiente de aprendizaje como promotor de las relaciones / interacciones del estudiante con su entorno

- El ambiente de aprendizaje como espacio de vivencia del conocimiento y como proceso pedagógico 


\section{Comportamiento como resultado de la influencia de los elementos del ambiente de aprendizaje}

Tal y como los autores Duval y Vayer (2015) lo afirmaron:

Todo lo que sucede o se expresa en la clase, a saber, las relaciones con los demás, el interés por las actividades, la participación en la acción, etc., depende en buena medida del modo en que se comprende la organización de las relaciones entre las personas y la organización de las estructuras materiales que subyacen a las interacciones sujetoentorno (p.27).

Por otra parte, nuevamente Fontana (1994) confirmó la idea de que los comportamientos inadecuados de los estudiantes en la clase, en muchas ocasiones no son propiciados por ellos, sino que hay otros componentes del ambiente que los pueden generar, inclusive cuando dichos comportamientos son extremos o propios de la personalidad del estudiante.

\section{Aprendizaje}

El autor Gagné (1975), expone que Aprender es el proceso básico por el cual adquirimos información del mundo exterior o interior para posteriormente trabajar con ella. Este proceso de asimilación de información mediante el cual se adquieren nuevos conocimientos, técnicas o habilidades. En este sentido, el aprendizaje consiste en adquirir, procesar, entender y aplicar una información que nos ha sido enseñada o que hemos adquirido mediante la experiencia a situaciones reales de nuestra vida. De allí que el aprendizaje pueda observarse tanto en los seres humanos como en los animales.

Ausubel, recalca el concepto de aprendizaje significativo para distinguirlo del repetitivo o memorístico y señala el papel que juegan las experiencias previas del alumno en la adquisición de nuevos conocimientos. Las experiencias o saberes previos es lo que el estudiante conoce sobre el tema y el grado de estructuración de lo que conoce. La significatividad sólo es posible si se relacionan los nuevos conocimientos con los que ya posee el sujeto.

En otras palabras, se entiende por aprendizaje al proceso de formar experiencia y adaptarla para futuras ocasiones, es decir aprender.

\section{Tipos de aprendizaje}

(Gagne, 1975), manifiesta que en la pedagogía como ciencia del estudio del aprendizaje, se distingue los siguientes tipos del mismo: 
- Aprendizaje receptivo: Aquellas dinámicas de aprendizaje en que el sujeto que aprende únicamente debe comprender, entender, el contenido para poder luego reproducirlo, sin que medie ningún tipo de descubrimiento personal.

- Aprendizaje por descubrimiento: Caso contrario al anterior, implica que el sujeto que aprende no reciba la información de manera pasiva, sino que descubra los conceptos y relaciones según su propio esquema cognitivo.

- Aprendizaje repetitivo: Se basa en la repetición del contenido a aprender, para fijarlo en la memoria. Es conocido como "caletre" o "aprender a la letra".

- Aprendizaje significativo: Aquel que le permite al sujeto poner en relación el nuevo contenido con lo que ya sabe, incorporándolo y ordenándolo para darle sentido según aprende.

- Aprendizaje observacional: Se basa en la observación del comportamiento de otro, considerado modelo, y la posterior repetición conductual.

- Aprendizaje latente: En este caso se adquieren nuevos comportamientos que permanecen ocultos (latentes) hasta que se recibe un estímulo para manifestarlo.

- Aprendizaje por ensayo y error: El aprendizaje conductista por excelencia, en el que se prueba una respuesta a un problema tantas veces como sea necesario para variar y encontrar la adecuada.

- Aprendizaje dialógico: Sostenido en el diálogo entre iguales, como hacían los antiguos filósofos griegos (de allí los Diálogos de Platón).

\section{Teorías del aprendizaje}

Según Skinner (1976) el aprendizaje es un cambio estable en la conducta o en la probabilidad de la respuesta que depende de los arreglos y contingencias ambientales. De modo que el individuo es absolutamente receptivo, pasivo, reactivo, dependiente fatalmente de las influencias externas. La respuesta depende del estímulo, el sujeto depende del objeto. Desde este enfoque, los conocimientos del sujeto son sólo la suma de asociaciones entre estímulos y respuestas, sin ningún tipo de organización o construcción estructural.

- Las teorías conductistas: Tales como el condicionamiento clásico de Pávlov, el conductismo de Skinner o el aprendizaje social de Bandura, se trata de un conjunto de teorías distintas que tienen en común la consideración del estímulo y la reacción como bases del aprendizaje. Un estímulo negativo desestimará una conducta, mientras que uno positivo la reforzará.

- Las teorías cognitivas: Posteriores a las conductistas, comparten con ellas algunos de sus principios, pero haciendo énfasis en el rol mucho más activo de quien aprende, ya que emplea en ello sus esquemas mentales y su enciclopedia de mundo, en base a lo que le resulta significativo. Ejemplos de ellas son el constructivismo de Piaget, el aprendizaje significativo de Ausubel y Novak, el cognitivismo de Merrill, o la Topología del aprendizaje de Gagné. 
- Las teorías del procesamiento de la información: Tales como el conectivismo de Siemens, ofrece una explicación sobre los procesos internos del aprendizaje basados en la interconexión y la idea de redes.

\section{El proceso de aprendizaje}

El Proceso de aprendizaje: De acuerdo a la teoría de Piaget (1969), el pensamiento es la base en la que se asienta el aprendizaje, es la manera de manifestarse la inteligencia, la cual desarrolla una estructura y un funcionamiento, ese mismo funcionamiento va modificando la estructura. La construcción se hace mediante la interacción del organismo con el medio ambiente.

Para aprender necesitamos de cuatro factores fundamentales: inteligencia, conocimientos previos, experiencia y motivación.

- A pesar de que todos los factores son importantes, debemos señalar que sin motivación cualquier acción que realicemos no será completamente satisfactoria. Cuando se habla de aprendizaje la motivación es el «querer aprender», resulta fundamental que el estudiante tenga el deseo de aprender. Aunque la motivación se encuentra limitada por la personalidad y fuerza de voluntad de cada persona.

- La experiencia es el «saber aprender», ya que el aprendizaje requiere determinadas técnicas básicas tales como: técnicas de comprensión (vocabulario), conceptuales (organizar, seleccionar, etc.), repetitivas (recitar, copiar, etc.) y exploratorias (experimentación). Es necesario una buena organización y planificación para lograr los objetivos.

- Por último, nos queda la inteligencia y los conocimientos previos, que al mismo tiempo se relacionan con la experiencia. Con respecto al primero, decimos que para poder aprender, el individuo debe estar en condiciones de hacerlo, es decir, tiene que disponer de las capacidades cognitivas para construir los nuevos conocimientos.

También intervienen otros factores, que están relacionados con los anteriores, como la maduración psicológica, la dificultad material, la actitud activa y la distribución del tiempo para aprender.

\section{El estudio en el proceso de aprendizaje}

(Meirieu, 2007) La cualidad de ser un buen o mal estudiante en función de su aprendizaje está en dependencia de su capacidad para asimilar los conocimientos, pero en ello influye un estudio eficaz, como lo son la convicción, la organización para desarrollar sus deberes escolares, la concentración, el poseer métodos propicios para lograr un correcto aprendizaje y formar hábitos de estudio. 
Por otra parte, algo muy importante para el desarrollo de un estudio eficaz son las condiciones del ambiente que te rodea y las personales. Desde el punto de vista ambiental el estudio se debe realizar en un lugar apropiado, es decir un lugar tranquilo y libre de distracciones, pues muchos de los estudiantes que presentan dificultades en el estudio generalmente expresan que carecen de un espacio adecuado para estudiar.

\section{Estrategias de estudio}

Al hablar de estrategias de estudios nos referiremos a lo que estudiar y que es aprender.

Son dos procesos activos, procesos complementarios pero diferentes. Estudiar es una actividad de aprendizaje intencional, intensivo y autorregulado, basado en un texto generalmente complejo y no familiar para el estudiante. Hernández y García (2005).

Cuanto más se conozca a sí mismo el estudiante y las diferentes técnicas que tiene a su disposición, más fácil le será seleccionar las más adecuadas a cada situación para crear sus propias estrategias de aprendizaje. Aprender a estudiar sacándole partido a las capacidades personales es muy parecido a un entrenamiento físico: hace falta voluntad, un buen entrenador y constancia. Si no conocemos la manera de hacerlo no llegaremos a tener buenos resultados. Hay que querer, pero también saber.

\section{Los Hábitos de estudio}

Para el autor Rondón (2001), precisa a los hábitos de estudio como conductas que manifiesta el estudiante en forma regular ante el acto de estudiar y que repite constantemente.

Otros Autores definen al hábito de estudio como: Una conducta o una cadena de conductas que son aprendidas por los estudiantes que tienen una altísima probabilidad de presentarse en un ambiente definido. También se le define como una cadena de conductas, que se adquieren en relación al estudio o en relación al logro relativo del dominio de contenido académico.

Los seres humanos exhibimos un sin número de comportamientos que ponemos en práctica en forma rutinaria. Son comportamientos que realizamos casi automáticamente sin que requieran de mucho esfuerzo consciente de nuestra parte. Esto es lo que comúnmente llamamos hábito. (Tovar, 2003)

\section{Mejorar los hábitos de estudio}

Los autores Kancepolski y Ferrante, (2006), manifiestan que los comportamientos poco saludables que desarrollamos alrededor de las actividades de estudio, y a los que a través de la repetición, invertimos una cantidad de tiempo considerable, pueden ser modificados favorablemente si activamente buscamos información adecuada y la ponemos en práctica. 
Requiriendo de un trabajo personal consciente, en donde se sustituyan hábitos poco saludables con aquellos que generen resultados más efectivos.

Esto requiere un trabajo personal consciente. Se debe sustituir un hábito o hábitos que no rinden los resultados deseados por otros que generen resultados más saludables y efectivos. En específico los objetivos son que: aumente el entendimiento y retentiva del material leído, logre mejorar sus calificaciones, haga el proceso de lectura y estudio uno más efectivo y agradable, se sienta mejor consigo mismo, sea más exitoso en llevar a cabo una tarea y tenga más tiempo para otras actividades igualmente importantes que aportan a desarrollar un ser humano feliz, saludable y funcional en la sociedad. (RIDE, 2006)

- Motivación y automotivación: La capacidad de motivación depende no sólo de que te gusten previamente las asignaturas y los profesores o profesoras, sino de ti mismo. Cuanto mejor te planifiques y te conozcas como estudiante mejor te motivarás.

- Lugar de estudio: Siempre el mismo, si puede ser con luz natural, en el caso contrario se debe usar la luz central y la focal a la vez para evitar el cansancio de los ojos, al pasar de zonas de oscuridad a zonas muy iluminadas.

- Tiempo de estudio: Cuando tienes trabajo por delante planificarlo te relajará pues te hará darte cuenta de que si cumples el horario te dará tiempo y podrás cumplir tus objetivos.

- Atención y concentración: En este punto se consideran de gran importancia los factores externos: Lugar de estudio, tiempo de estudio, ruidos o molestias del exterior y los factores internos: Preocupaciones, falta de concreción en los objetivos, cansancio, nerviosismo, etc. Más difíciles de controlar, pero no imposible.

\section{Técnicas de estudio}

Las técnicas de estudio son un conjunto de procedimientos que nos permiten mejorar la calidad, eficacia y eficiencia de nuestros estudios y aprendizajes en un área determinada constituyéndose en un proceso de adquisición de habilidades y conocimientos, que se produce a través de la enseñanza, la experiencia o el estudio. (González, R. \& González J.A. , 2005)

\section{Flashcards}

Conocidas en español como tarjetas de aprendizaje, tarjetas educativas, tarjetas de estudio, tarjetas didácticas, tarjetas de memorización o tarjetas mnemotécnicas son tarjetas que contienen palabras, imágenes, símbolos o números en uno o ambos lados y se usan para adquirir conocimientos al memorizar su contenido mediante el repaso espaciado del conjunto de tarjetas. Tienen como objetivo estimular la memoria y hacer que el estudiante piense y busque la información que ha guardado en su cerebro de manera rápida y práctica.

Estas tarjetas pueden ser de papel o digitales. Actualmente con el avance de la tecnología y el consumo de los medios digitales, se puede aprovechar esta técnica de estudio en Internet y aplicaciones móviles, lo cual da más ventajas como la posibilidad de compartir los materiales 
de estudio con más personas, utilizar las tarjetas en modo de prueba y filtrar las tarjetas que aún no se aprenden. (Cohn, 2013)

\section{Subrayar}

Según Cuenca (2000), "Esta técnica tiene por objeto resaltar, mediante el subrayado con trazos diferentes o colores, las palabras del texto que contienen o representan las ideas o conceptos fundamentales y los enlaces (y, ni, pero...) que puedan favorecer la comprensión o estructura del texto escrito" (p.211). Pueden destacarse con una línea, horizontal o vertical, los enunciados que expresen las ideas principales de un texto. Con el subrayado se busca que el lector extraiga del texto las ideas principales y las secundarias del autor, para lo cual se pueden utilizar diferentes señales y colores.

\section{Notas o anotaciones al margen}

Se realizan siempre después de la pre lectura o lectura rápida de un texto, estas anotaciones devienen de las ideas principales que se van descubriendo en cada párrafo, para ello se lee párrafo por párrafo con ayuda de un diccionario, cuando sea necesario. Se puede elaborar las notas al margen haciendo preguntas, como por ejemplo: ¿De qué trata este párrafo? ¿Qué dice realmente?, y las respuestas se escriben en el margen izquierdo del texto.

Es importante destacar que una anotación al margen no es la transcripción de la idea principal del párrafo analizado; tampoco un conjunto de palabras sin sentido lógico. Una buena anotación al margen consiste en sintetizar el párrafo analizado. Este tipo de trabajo nos ayuda a realizar el estudio más activo y agradable y comprender mejor el tema para posteriormente realizar el subrayado y el esquema, asimismo nos permite plantear los subtítulos del tema.

\section{El resumen}

Es una de las técnicas más utilizadas por el estudiante pues orienta el sentido y comprensión del texto que analiza. Muchos aún consideran que el resumen es la sumatoria de las ideas principales de un texto ordenadas con lógica y coherencia, lo cual es válido. Según Bernardo (2000):

... consiste en la reducción de un texto respetando su sentido y empleando las palabras del autor (...) como su objetivo es reducir el texto a la información fundamental, con respeto fiel a las palabras del autor, esta técnica es considerada como una de las más sencillas y, por tanto, la más apropiada en las primeras etapas del estudio. (p.47).

Un buen resumen no debe exceder más del 30\% de la extensión del texto original y debe reflejar las ideas más importantes expresadas en forma personal, con las propias palabras.

\section{La síntesis}

De acuerdo a lo citado por Bernardo (2000): 
...tiene por finalidad exponer brevemente las ideas fundamentales de un texto, pero con palabras propias de quien lo realiza. Esto exige la transformación de lo leído en frases comprendidas y asimiladas, poniendo en juego el pensamiento reflexivo, la elaboración personal y el uso de un lenguaje propio... (pp. 47-48).

En esta definición radica la importancia de la síntesis y su relación con la competencia argumentativa del estudiante.

\section{Toma de notas o apuntes}

Se considera una actividad compleja que implica la escucha, el análisis, la síntesis, la selección, la organización, la escritura y que depende de diversos factores. Así, por ejemplo se sugiere que el desarrollo de esta actividad está en función de las metas de los estudiantes, de la relevancia del material, de los conocimientos y experiencias previas, de las concepciones personales sobre el aprendizaje o de los estilos de exposición de los docentes o conferencistas. Para ello se tomará en cuenta lo siguiente: El conocimiento previo del estudiante, la planificación de la toma de notas y la supervisión y revisión durante la toma de notas y el sistema Cornell.

Tabla N1, Notas tomadas en el aula

\begin{tabular}{|c|c|}
\hline Columna de indicios & Notas tomadas en el aula \\
\hline & Aprendizaje significativo \\
\hline \multirow[t]{2}{*}{ ¿Qué es? } & $\begin{array}{l}\text { Es la relación que existe entre el conocimiento previo y el nuevo } \\
\text { conocimiento. }\end{array}$ \\
\hline & Conocimiento organizado \\
\hline \multirow[t]{3}{*}{ Condiciones } & Buena disposición \\
\hline & Estructura cognoscitiva entre conocimiento previo y nuevo. \\
\hline & De representaciones (significado de palabras) \\
\hline Tipos & De proposiciones (significado de ideas) \\
\hline
\end{tabular}

Fuente: Casas, E. y Garay, L. (2006). Técnicas de estudio e investigación. Lima: UIGV.

\section{Organizadores de la información}

(Delgado, 2007) Según investigaciones académicas, la realización de mapas conceptuales y/o mentales, la esquematización y organización de estos ayudan al estudiante a comprender de manera práctica y sencilla un tema en específico. De esta manera, le ayudan a estudiar, organizar ideas, repasar para los exámenes y extraer buenas notas. 
El mapa conceptual fue ideado por Joseph D. Novak a mediados de los años sesenta del siglo $\mathrm{XX}$; es "una representación gráfica, esquemática o fluida, donde se presentan los conceptos relacionados y organizados jerárquicamente" (Ontoria, 1996, p.39). Como estrategia de aprendizaje, el mapa conceptual hace que el estudiante elabore contenidos a través de la elección de conceptos, decide la jerarquía y las relaciones entre ellos, al mismo tiempo que estimula el desarrollo de la creatividad, posibilita el trabajo en equipo y exige esfuerzo intelectual, Ontoria señala que: "La elaboración de mapas conceptuales fomenta el pensamiento reflexivo, la creatividad y el espíritu crítico. El pensamiento reflexivo es un quehacer controlado, que implica llevar y traer conceptos, uniéndolos y volviéndolos a separar" (Ontoria, 1996, p.42)

Los Mapas Conceptuales (MMCC) son instrumentos que permiten construir el conocimiento y que responden a las siguientes características establecida por su creador J. Novak, estas son:

- Jerarquización: En los MMCC los conceptos están dispuestos por orden de importancia o de "inclusividad". Los MMCC se leen de arriba hacia abajo.

- Estructura proposicional: Los MMCC expresan explícitamente las relaciones más relevantes entre un conjunto de conceptos. Al construir un $\mathrm{MC}$, debe tenerse cuidado de que cada dos conceptos enlazados con sus palabras de enlace forman una unidad de significado.

- Pregunta Enfoque: Para delinear el contexto de un MC se necesita de una PREGUNTA ENFOQUE, esto es, una pregunta que claramente especifique el problema o asunto que el $\mathrm{MC}$ debe tratar de resolver.

- Enlaces Cruzados: El MC necesita de relaciones o enlaces entre conceptos de diferentes segmentos o dominios del MC. Los enlaces cruzados nos ayudarán ver cómo un concepto en un dominio de conocimiento representado en el mapa está relacionado con un concepto en otro dominio expresado en el mapa. Un enlace cruzado generalmente representa un salto creativo por parte del constructor del mapa.

\section{Organizadores del Pensamiento.}

Mediante esta técnica se busca obtener una visión totalizadora del tema de estudio, facilitándonos la memorización y el repaso del mismo, permitiendo que el que lo usa perciba la información expresada sintéticamente.

\section{Los Esquemas}

El esquema es la síntesis estructurada de un concepto o contenido, de un tema, atendiendo a sus características más significativas e ideas importantes, se desagrega en categorías. Conceptos que a su vez contienen subcategorías o subconceptos. Podemos definirlo como la representación gráfica de un concepto atendiendo a sus características más significativas.

\section{Estructura del esquema}

Según Castillo y Polanco (2005) la estructura sería la siguiente: 
a. Elementos: Título del esquema en mayúscula, apartados sectoriales o estructurales que conserven las divisiones y subdivisiones.

b. Tipo de letra: Se realiza según la importancia de la idea. El título va en mayúscula y luego podría ir en mayúscula las ideas fuerza y principales mientras que las ideas secundarias van en minúsculas. Esta regla es opcional.

c Distribución de las ideas: Se puede dar:

a. De arriba abajo (esquema de desarrollo o vertical)

b. De Izquierda a derecha (esquema de llaves, de barras o sinóptico

d. Signos de diferenciación en la distribución de las ideas: Se pueden colocar números, letras, signos, códigos; todo lo que posibilite sintetizar información.

e. Presentación: Se maneja los siguientes criterios:

a. Limpieza y claridad

b. Utilización generosa del espacio

c. Signos, códigos, letras y texto utilizados correctamente, según las normas.

d. Jerarquía y coherencia.

\section{Tipos de esquemas}

Existe una vasta clasificación de esquemas, su empleo dependerá del tipo de texto trabajado y de la utilidad que le quiera dar el lector. A continuación, detallamos algunos:

- Esquema de llaves: Llamado también cuadro sinóptico, se emplea cuando el tema exige muchas subdivisiones. Es sintético, por lo tanto, se utiliza más títulos que contenidos descriptivos. Se expresa de izquierda a derecha, tiende a ser abarcativo en esa dirección. Se realiza a partir de una idea principal (expresada en mayúscula) hasta llegar a subdivisiones de menor importancia, (expresadas en minúscula) existiendo congruencia entre todas sus dependencias (El subrayado constituye el instrumento eficaz para ello).

- Diagrama: Es el esquema cuyo diseño es el más sintético de todos. La presentación gráfica permite apreciar las relaciones entre conceptos e ideas fundamentales con las secundarias. Son especialmente útiles para representar clasificaciones bien definidas como organigramas, genealogías, jerarquías, relaciones entre órganos e identidades, etc. Existen diversos tipos de diagramas entre las cuales podemos mencionar: diagrama de flujo, diagrama de proceso, diagrama de arco, diagrama causa efecto, diagrama pirámide, diagrama circular, etc.

- Cuadro comparativo: Consiste en un cuadro de doble entrada que permite organizar la información de acuerdo con unos criterios previamente establecidos. La finalidad principal es establecer las diferencias entre los conceptos que se tratan. Pueden emplearse para expresar analogías, diferencias respecto a conceptos, teorías. Se elabora teniendo presente la correspondencia entre los elementos de análisis.

- Listas de palabras en inglés: Para lograr mantener una comunicación fluida en inglés es importante conocer de manera práctica vocabulario en inglés. Por esta razón es importante que realice un listado de palabras que más necesite usar en inglés profesional, si las escribe 
y las repasa diariamente podrá mejor así su vocabulario, pronunciación y comunicación en inglés.

Para finalizar, recuerde que la práctica hace al maestro. Efectivamente hoy está a tiempo de poner en práctica estás técnicas de estudio y mejorar así su proceso de aprendizaje en inglés profesional, ; ahora es el momento! (Martinez, 2018)

\section{Idioma ingles}

El inglés es la principal lengua extranjera enseñada en el sistema educativo nacional, según las cifras oficiales en el periodo de 2010 a 2011, cuenta con un total de 34568 escuelas con 316 440 maestros en escuelas públicas en todos los sistemas (SNIE, 2010). Si bien no hemos ubicado cifras exactas del número de maestros de inglés en secundarias, por ejemplo, se infiere por un comentario emanado de la Dirección General de Formación Continua de Maestros en Servicio (DGFCMS), que existen cerca de 30 mil profesores de la asignatura de inglés de secundarias públicas, en todas sus modalidades. Agreguemos a este panorama el hecho de que en años recientes se ha iniciado un movimiento hacia la generalización de la enseñanza del inglés en primarias en la educación pública.

De acuerdo a lo explicado por Álvarez (2010), el aprendizaje de una segunda lengua resulta indispensable en la sociedad actual ya que ayuda a los alumnos a desarrollar una identidad cultural global, necesaria para aprender a vivir en una sociedad en la que continuamente se establecen relaciones con personas de diferentes nacionalidades y culturas.

\section{La importancia del aprendizaje del inglés desde una edad temprana}

Noam Chomsky (1965, citado en Sarmiento, 1996) afirma que los seres humanos tenemos la capacidad innata para adquirir el lenguaje que no se aplica únicamente al aprendizaje de la lengua materna sino a todas las lenguas y que la función que cumple el ambiente que rodea al niño no es la de enseñar directamente, sino la de "activar la capacidad innata y convertirla en competencia lingüística” (Sarmiento, 1996, p. 292). Se justifica esta idea al saber que nunca es demasiado pronto para exponer a un niño a la segunda lengua en razón de que desde que nace presenta la capacidad para aprenderla.

Fleta (2006) indica que en el caso de los niños que establecen contacto continuo con dos lenguas desde que nacen, las van adquiriendo a la vez y normalmente llegan a tener un nivel nativo en las dos lenguas, son los bilingües pre-coses o monolingües en dos lenguas.

\section{El aprendizaje del idioma inglés en la enseñanza superior}

"La enseñanza del inglés en cualquier parte del mundo, en la actualidad, es vista como una necesidad y una urgencia. No importa en qué continente te encuentres o la edad que tengas, el 
hecho es que para cualquier persona es indispensable tener los respectivos conocimientos de la lengua inglesa". (Chango, 2009)

Es la educación superior el último escalón y la última opción desde el punto de vista escolar para alcanzar un buen nivel en este idioma. En diversas universidades del mundo, cuyos estudiantes no poseen como lengua materna el inglés, se ha potencializado y se continúan desarrollando estructuras y mecanismos para enseñar este idioma.

Al igual que la mayoría de los países de habla hispana, Ecuador vive una situación lingüística diversa y compleja porque no solo cuenta la enseñanza de lenguas extranjeras sino también en la de las lenguas nacionales (lenguas originarias) que, tradicionalmente se han abordado de forma separada. El español (lengua materna), muy poco se lo toma en cuenta para la enseñanza de habitantes extranjeros que residen en el país (Mina, 2007).

"En la educación superior se espera que los estudiantes recién graduados sean capaces de comprender textos complejos que se encuentren dentro de su área de conocimiento y puedan expresarse con cierto grado de fluidez cuando se comuniquen con otras personas en inglés, por tal razón las Instituciones de Educación Superior deben garantizar el cumplimiento de un número de horas básicas y la calidad en las cátedras que son impartidas". (Ministerio de Educación Nacional de Colombia, 2005)

Para garantizar esto, la enseñanza superior en varios países organiza una estrategia que puede comprender diversas asignaturas obligatorias de inglés, así como un examen de estricta aprobación para obtener el título. Por otro lado, puede establecer convenios con instituciones especializadas en la enseñanza de este idioma, e insertar a los estudiantes en cursos.

Sin embargo, todavía queda mucho por hacer en cuanto a la formación en las universidades. Según Pedro Hernández (2012), Director de Relaciones con Órganos Colegiados de la Asociación Nacional de Universidades e Instituciones de Educación Superior, (ANUIES) de México: "En América Latina, la enseñanza y el aprendizaje de por lo menos un idioma diferente al español es, todavía, un reto en la formación profesional, ya que existen aún dificultades, o se avanza lentamente, para incorporar en los programas de estudio la enseñanza de idiomas y lograr que los estudiantes a su egreso tengan el conocimiento y dominio de al menos una lengua extranjera". (Sprachcaffe., 2017)

Según investigaciones científicas, estudiar inglés o aprender cualquier idioma diferente al nativo, puede generar ciertos estímulos en el cerebro que ayudan a mejorar las habilidades en otras áreas como son la creativa, la resolución de problemas, el razonamiento o la habilidad mental". (Bonilla, 1195)

Sin embargo, se conoce que "Hoy en día cualquier investigador o profesional que quiera estar al día o acceder a libros especializados necesita irremediablemente saber inglés para estar 
informado, porque precisamente el 75\% de la bibliografía científica está en inglés". (Ortiz, 2013)

\section{Adquisición de un Segundo Idioma}

Diferentes estudios han buscado entender cómo se da este proceso de adquisición, y si este proceso se da de forma consciente o inconsciente, para poder entender por qué los estudiantes alcanzan diferentes niveles de conocimiento del segundo idioma, y cómo ello afecta en su habilidad de comunicación (Gass, S. \& Selinker, L. , 2008). Estos trabajos han dado gran aporte al surgimiento de nuevas metodologías.

De acuerdo con Rojas y Garduño (s.f.), la adquisición de una lengua extranjera es un proceso de aprendizaje que sucede la mayor parte del tiempo en un ambiente de formación académica, y que se da en un lugar en el cual no se usa el idioma. Es decir, que la adquisición de una segunda lengua se da luego de que el individuo ha aprendido su lengua materna.

Además, el proceso mediante el cual el estudiante adquiere el idioma inglés, difiere en gran medida de un proceso de aprendizaje, ya que este último es un proceso consciente. Para poder explicar este proceso es necesario conocer a detalle aquellas teorías que proponen y explican cómo se da este proceso de adquisición, las cuales se considerarán en el siguiente apartado.

\section{El rol del profesor}

El profesor hace tiempo era considerado como el único y verdadero dueño del conocimiento, y cuya función era únicamente transmitir este (Hernández, E. \& Valdez, S., 2010). Sin embargo, en la actualidad y dado los enormes avances en la ciencia y tecnología ese rol ha cambiado, pasó de ser un rol protagónico y muchas veces imponente a un rol accesible y que cumple con la función de guiar y mejorar el proceso de enseñanza aprendizaje del idioma Inglés, valiéndose para ello de un sinnúmero de estrategias orientadas a desarrollar la parte comunicativa del estudiante (Guevara, 2000)

El aprendizaje del idioma inglés requiere docentes totalmente capacitados en cuanto a la metodología

apropiada para desarrollar un correcto proceso de enseñanza aprendizaje del idioma, que tenga la capacidad de reconocer los diferentes tipos de estudiantes, sus formas de aprendizaje, sus problemas en el aprendizaje, etc. (Juan, A. \& García, I., 2012)

De acuerdo con Juan y García (2012), dentro del aula de clase los estudiantes harán uso del idioma inglés siempre y cuando el profesor considere los siguientes aspectos:

La Planificación de los procedimientos que requieren el uso de la segunda lengua, de acuerdo con el nivel de los alumnos y teniendo en cuenta que, por lo general, comprenden más de lo que son capaces de producir. 
Expresarse de la forma más clara y sencilla posible. Reforzar visualmente las explicaciones y las correcciones por medio de gestos, dibujos, objetos, el uso de la pizarra, etc.

- Acostumbrar a los alumnos a que empleen fórmulas idiomáticas en inglés. La enseñanza y la promoción del uso de términos genéricos mejoran su capacidad de comunicación.

- Familiarizar a los alumnos con las expresiones más comunes que se utilizan en el aula y su uso frecuente y coherente. Promover la capacidad de los alumnos para inferir el significado por medios lógicos como el contexto y las asociaciones. (p. 7).

Al considerar cada uno de los aspectos mencionados anteriormente, se logrará brindar las condiciones adecuadas que permitan el uso permanente y la práctica diaria del inglés, además se logrará motivar al estudiante dentro de un aprendizaje permanente.

\section{El rol de estudiante}

Representa el componente más importante dentro del proceso de enseñanza y aprendizaje del idioma Inglés dado que una vez finaliza el mismo, serán ellos en quienes se podrá verificar los resultados del aprendizaje; es decir, el grado de competencia comunicativa que ha logrado alcanzar el estudiante al final del curso.

El rol del estudiante es uno de los principales cambios que se ha logrado en la enseñanza del idioma. Son ellos quienes desempeñan un rol más activo ya que serán los responsables del nivel de desarrollo de las habilidades del idioma y son ellos quienes deberán una mayor iniciativa dentro del aula de clase (Ordorica, 2010)

Para los estudiantes que estudian Inglés como lengua extranjera uno de los espacios de aprendizaje más importantes es el aula de clase, dentro de la cual se desarrolla su proceso de aprendizaje y que será el espacio propicio, en el cual se darán las condiciones necesarias para que pueda tener la oportunidad de comunicarse en el idioma Inglés. (Juan, A. \& García, I., 2012)

\section{Referencias Bibliográficas}

Ausubel, D. P. (2000). Adquisición y Retención del Conocimento. Una perspectiva cognitiva. Nueva York : Columbia.

Bernardo, J. (2000). Hacia una enseñanza eficaz. Madrid: Ediciones RIALP S.A.

Bonilla, C. (1195). Importancia social de los Ambientes Modernos de Aprendizaje. Revista Kinesis.

Cantero, V. (2011). La enseñanza de segundas lenguas a través de tareas: una propuesta didáctica para el $1^{a}$ de ESO Bilingüe. Tendencias Pedagógicas, (17), 134-156. 
Castillo, S. \& Polanco, L. (2005). Enseña a estudiar... aprende a aprender. Madrid: Pearson: Prentice Hall.

Chango, J. (24 de 02 de 2009). Obtenido de Importancia del ingles en la educacion: Disponible en https://es.slideshare.net

Cohn, M. (2013). Helping Your Teen-age Student: What Parents Can Do to Improve Reading and Study Skills. . Dutton. .

Colombia, M. d. (2006). Formar en Lenguas extranjeras: El reto , lo qu necesitamos saber y hacer. Colombia: Espantapajaros.

Conceptos., D. (22 de 03 de 2019). deconceptos.com. Obtenido de http://deconceptos.com/cienciassociales/cuestionario.

Contreras, O. (2012). Stephen Krashen: sus aportes a la educación bilingüe. Rastros Rostros, 14, (27), 123-124.

Coombs, P. H. (1985). a Crisis Mundial en la Educación. Perspectivas actuales. Madrir: Santillana.

Correa, M. (2003). Hábitos de Estudio y Tarea en Casa. . USA.: Ediciones de la Universidad de Illinois.

Delgado, A. (2007). Guía para el desarrollo del pensamiento creativo (2a. ed.). Lima: Ministerio de Educación del Perú.

Duval, P, \& Vayer, R. . (1993). Una ecología de la escuela: La dinámica de las estructuras materiales.

Duval, P, \& Vayer, R. (2015). Una ecología de la escuela: La dinámica de las estructuras materiales. Madrid: Paios.

Ecured. (155 de 03 de 2019). www.ecured.cu/Ambiente_educativo. Obtenido de https://www.ecured.cu/Ambiente_educativo

Fontana, D. (1994). El control del comportamiento en el aula. Barcelona: Paidós.

Freire, P. (1980). Pedagogía del Oprimido, Siglo XXI, . Madrid.

Gass, S. \& Selinker, L. . (2008). Second Language Acquisition: An introductory course. . Francia: Routledge.

González, R. \& González J.A. . (2005). Estrategias y Técnicas de Estudio. Madrid: Pearson Educación.

Guevara, J. (2000). La enseñanza del Inglés en la era digital. Revista de Ciencias Humanas, 16.

Hernández, E. \& Valdez, S. ( 2010). El papel del profesor en el desarrollo de la competencia intercultural. Algunas propuestas didacticas. 
Hernández, F. (2000). Los métodos de enseñanza de lenguas y las ateorias de aprendizaje. Revista de Investigacion e innovacion en la clase de idiomas , (11) 141-153.

Juan, A. \& García, I. (2012). Los diferentes roles del profesor y los alumnos en el aula de lenguas extranjeras". . Didacta, 21, (38).

Latina, I. F. (2010). ambafrance-mx.org/espanol/cultura-ycooperacion/instituto-frances-deamerica-706/cooperacion-linguistica-y-712/article/cooperacion-linguistica-y. Obtenido de http://www.ambafrance-mx.org/espanol/cultura-ycooperacion

Littlewood, W. (1992). La enseñanza de la comunicación oral. . Barcelona: Paidós.

López, S. N. (2005). Modelo de teoría y diseños didácticos . Medellin : adryvirtual1.blogspot.com. Obtenido de Modelo de teoría y diseños didácticos “ Colombia Medellín 2005 $\square$ Modelos pedagógicos “adryvirtuall.blogspot.com: https://www.ecured.cu/Ambiente_educativo

Loughlin, C. \& Suina, J. (2002). El ambiente de aprendizaje: diseño y organización. Madrid:: Morata.

Martinez, P. (21 de 10 de 2018).

Meirieu, P. (2007). Cuadernos de Pedagogía.

Mina, V. C. (2007). El español escrito de estudiantes bilingües en la Universidad Intercultural del estado de México. México: UAEM.

Muñoz, A. (2010). Metodologías para la enseñanza de lenguas extranjeras Hacia una perspectiva citica. Revista Universidad EAFIT, , 46, (159), 71-85.

Muñoz, C. B. (2002). Aprender idiomas. Barcelona. Ediciones : Paidós S. A.

Novak, J. \& Gowin, B. (1988). Aprendiendo y aprender. Barcelona:: Ediciones Martínez Roca, S.A.

Ontoria, A., Molina, A. \& De Luque, A. (1996). Los mapas conceptuales en el aula. Buenos Aires: Editorial Magis.

Ordorica, D. (2010). Motivación de los estudiantes universitarios para estudiar Inglés como lengua extranjera. Leaa. . Revista Electrónica. 3, (2).

Piaget, J. (1969). Psicologia y Pedagogia: Teorias del aprendizaje . Barcelona: Planeta.

RIDE. (2006). Hábitos de Estudio y rendimiento academiao. Cao estudiantes de licenciatura en administracion . Revista iberoamericana desarrollo educatvo., 10.

Rojas, C. (04 de 08 de 2016). https://eslbyltaonao/entorno-educativo/. Obtenido de https://eslby1 taonao/entorno-educativo/

Rojas, F. \& Garduño, G. (s.f.). (05 de 2006). Adquisición de una lengua segunda desde el punto de vista la lingüística. Obtenido de elinguistica.azc.uam.mx/no005/06.htm.: https//relinguistica.azc.uam.mx/no005/06.htm. 
Rondón, C. (2001). Internalidad y Hábitos de Estudio. Tesis de Maestría. . Caracas. Venezuela.: Universidad Pedagógica Experimental Libertador. .

Salle, L. (20 de 03 de 2019). http://www.colombiaaprende.edu.co/html/productos/1685/w3article-288989.html)

Obtenido

de http://www.colombiaaprende.edu.co/html/productos/1685/w3-article-288989.html)

Sierra, I., \& Carrascal, N. (2008). La gestión de ambientes de aprendizaje y el desarrollo de Competencias. Colombia: Alfa Editores, Comunicación Estratégica.

Skinner, B. (1976). Influencia de la teoria con el aprendizaje. Estados Unidos : Pensilvania.

Sprachcaffe. (15 de 03 de 2017). ¿Por qué estudiar inglés? España. Obtenido de sprachcaffe.com: http://www.sprachcaffe.com

Tovar, A. (2003). Efectos de los Hábitos de Estudio en el Rendimiento Académico de los Alumnos de la Escuela de Formación Deportiva "Germán Villalobos Bravo. Caracas, Venezuela.

UNESCO. (2002). Estudio cualitativo de Las escuelas con resultados destacables en siete países Latioamericanos . Chile: Calidad Educativa.

www.significados.com/aprendizajel. (22 de 03 de 2019). Obtenido de https://www.significados.com/aprendizaje/ 


\section{PARA CITAR EL ARTÍCULO INDEXADO.}

Colcha Guashpa, E., Herrera Andrade, Z., Barragán Murillo, R. de los Ángeles, Guano, D., \& Salazar Calderón, E. (2019). Entorno educativo y aprendizaje, estrategias de estudio en el idioma ingles. Ciencia Digital, 3(2.6), 349-369. https://doi.org/10.33262/cienciadigital.v3i2.6.571

\section{Ciencia \\ Ligital \\ Editorial}

El artículo que se publica es de exclusiva responsabilidad de los autores y no necesariamente reflejan el pensamiento de la Revista Ciencia Digital.

El artículo queda en propiedad de la revista y, por tanto, su publicación parcial y/o total en otro medio tiene que ser autorizado por el director de la Revista Ciencia Digital.
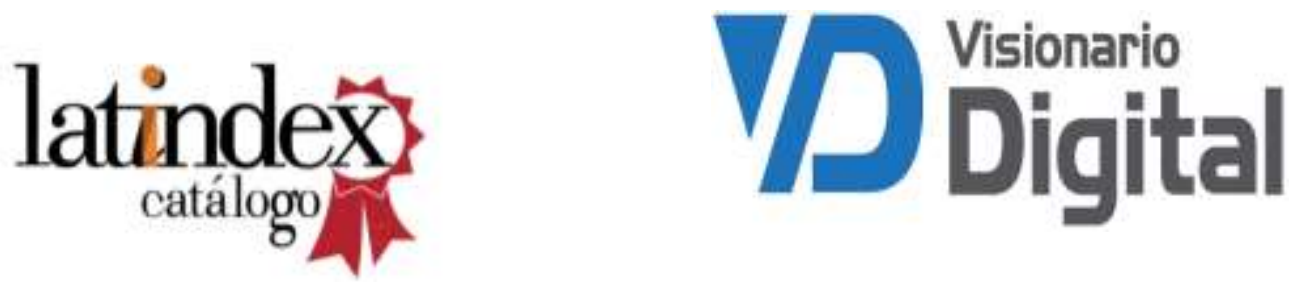